\title{
Why Evaluate the Sunscreen Active Oxybenzone (Benzophenone-3) for Carcinogenicity and Reproductive Toxicology or Consider it Unsafe for Human Use?
}

\author{
DiNardo JC, $\mathrm{MS}^{1}$ and Downs $\mathrm{CA}, \mathrm{PhD}^{2}$
}

\author{
${ }^{1}$ Research Scientist Emeritus, Vesuvius VA 24483, United States. \\ ${ }^{2}$ Executive Director, Haereticus Environmental Laboratory, \\ Clifford, VA 24533, United States.
}

*Correspondence:

Joseph C DiNardo, MS, Research Scientist Emeritus, Vesuvius VA 24483, United States.

Received: 11 May 2019; Accepted: 22 June 2019

Citation: DiNardo JC, Downs CA. Why Evaluate the Sunscreen Active Oxybenzone (Benzophenone-3) for Carcinogenicity and Reproductive Toxicology or Consider it Unsafe for Human Use?. Dermatol Res. 2019; 1(1); 1-3.

\section{Keywords}

Carcinogenicity, Reproductive Toxicology, DNA, FDA.

\section{Reason 1}

Oxybenzone passes through human skin and enters into blood plasma at levels between 339 and 419 times greater than the level for toxicological concern that FDA considers necessary to demonstrate human safety.

On February 26, 2019, the Food \& Drug Administration (FDA) published an update to the Sunscreen Drug Products for Overthe-Counter Human Use in the Federal Register [1], requesting additional information on 12 petroleum-based sunscreen actives (cinoxate, dioxybenzone, ensulizole, homosalate, meradimate, octinoxate, octisalate, octocrylene, padimate $\mathrm{O}$, sulisobenzone, oxybenzone and avobenzone). This is because the public record does not currently contain sufficient data to support that these drugs are Generally Recognized as Safe and Effective (GRASE). The FDA went on to conduct and publish a small clinical trail [2] to determine whether four of these drugs (avobenzone, oxybenzone, octocrylene, and ecamsule) absorb into the systemic circulation and whether they would exceed the FDA level for toxicological concern $(0.5 \mathrm{ng} / \mathrm{mL})$. The data collected demonstrated that all four sunscreen drugs absorbed through the skin and into the blood, and accumulated above the $0.5 \mathrm{ng} / \mathrm{mL}$ level within the first day (4 applications) of the study. Oxybenzone exceeded the level of concern after the first application demonstrating an overall maximum plasma concentration between 169.3 and $209.6 \mathrm{ng} / \mathrm{mL}$ for concentrations ranging from $4 \%$ to $6 \%$ in products, respectively.

\section{Reason 2}

There is data demonstrating that oxybenzone causes damage to DNA, is mutagenic and is structurally similar to benzophenone a known carcinogen.
In vitro, oxybenzone demonstrated DNA damage under a number of test conditions including sister chromatid exchange/ chromosomal aberrations in Chinese Hamster Ovary cells and was found to be weakly mutagenic in salmonella with metabolic activation [3]. Two metabolites of oxybenzone, benzophenone and benzophenone-1, have also been found to be mutagenic in salmonella [3]. Oxybenzone, benzophenone-1 and 4-hydroxybenzophenone (another oxybenzone metabolite) have also been shown to stimulate cellular proliferation of human breast cancer cells and may also have a similar effect on prostate and lung cancers [3]. Pre-clinical data from a 2 and 13 week topical and ingestion studies on oxybenzone in mice and rats conducted by the National Toxicology Program (NTP) demonstrated consistent toxicological findings that included decreases in epididymal sperm density, lengthened estrous cycle, and increased liver and kidney weights [4]. Similar findings were noted in a 2-year carcinogenicity study conducted by NTP on benzophenone [5]. In fact, the latter study was used by FDA to ban benzophenone as a food additive and as a plasticizer in rubber articles intended for repeated use in contact with food in October 2018 [6]. The International Agency for Research on Cancer (IARC) and California Prop 65 also identify benzophenone as a carcinogen $[7,8]$. Lastly, a product containing $6 \%$ oxybenzone and $10 \%$ octocrylene was evaluated for benzophenone content and was found to contain 75.95 parts per million of the carcinogen [3].

\section{Reason 3}

There are over 175 scientific articles, mostly published in peer review journals that relate to some form of toxic adverse reaction occurring after exposure to oxybenzone.

A literature compilation, categorized by topic, can be found in a submission to the FDA that lists $175+$ publications written by hundreds of scientists from around the world [3]. From these studies, 
it is demonstrated that oxybenzone is chemically aggressive, forming free radicals as well as reacting with chlorine, bromine and ozone to form toxic byproducts in pools, spas and tap water. Environmentally, the chemical bioaccumulates and biomagnifies and should be considered a water and food contaminant since it can be measured in virtually all bodies of water and in many edible fish. It demonstrates general toxicity (acute and sub-chronic) in addition to mutagenic, cytotoxic, genotoxic, neurotoxic, numerous endocrine disruption activities, proliferates human breast/lung/ prostate cancer cells, produces phototoxic, contact/photo-contact allergic as well as contact urticaria, contact mediated anaphylaxis and can be found in the urine, blood and breast milk of numerous aquatic and terrestrial species including humans.

\section{Reason 4}

There are data demonstrating that oxybenzone can act as an endocrine disrupting chemical in many different types of species including humans and can cause variations and/or anomalies in the next generation.

With respect to available published reproductive toxicity data, oxybenzone has a molecular weight of $228.2 \mathrm{~g} / \mathrm{mol}$, which is small enough to allow it to pass through skin and placenta barriers $[9,10]$. Oxybenzone has been shown to produce endocrine disrupting effects in coral larvae, harlequin fly, several types of fish with adult and juvenile males becoming feminized/developing egg proteins, reducing mature spermatozoa in testicular tissues, decreases agonistic behavior in Siamese fighting fish and can reduce the number of eggs produced in females [3]. Additionally, the 2 and 13-week NTP studies in rats and mice noted above, demonstrated consistent toxicological findings that included decreases in epididymal sperm density and lengthened estrous cycles [4]. In human offspring, oxybenzone has been identified as decreasing fat mass during neonatal exposure, decrease body mass index in 5-6 month old infants, decrease birth weight in girls, increase birth rate in boys, lower abdominal circumference and femur length, lower testosterone levels in boys, altered pubertal development and timing, delayed breast development in girls and Hirschsprung's Disease [3] (Figure 1).

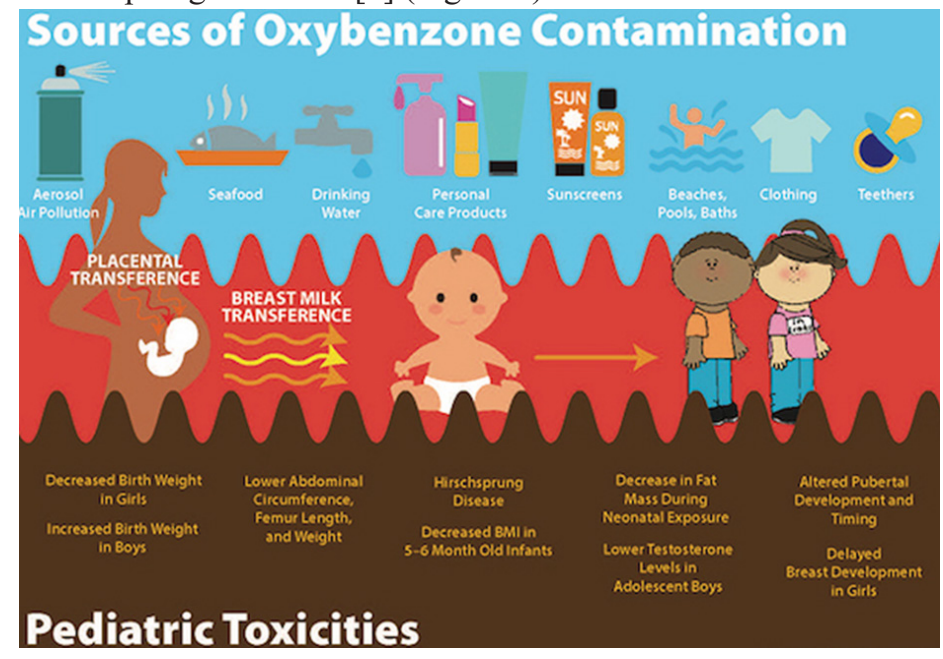

Figure 1: Oxybenzone Pediatric Toxicities.

\section{Reason 5}

Industry is stating that sunscreens are safe and effective for their intended use. This is in complete disagreement with the preponderance of the scientific literature and the guidelines set forth by the people (FDA) who are charged with protecting the consumer from harm.

The Personal Care Products Industry (which sells over $\$ 10$ billion a year in sunscreen products) is trying to convince FDA that these chemicals do not require additional testing based on what they call a safe history of use in humans and the protection they afford consumers against skin cancer. Neither statement can be substantiated since there are hundreds of scientific papers in the literature clearly identifying various toxic reactions in many species, including humans [3]. With respect to sunscreens and their ability to inhibit skin cancer, IARC stated in 2000 that "no conclusion can be drawn about the cancer preventive activity of sunscreens against basal cell carcinoma and melanoma" [11]. This conclusion was supported by a 2019 publication [12] that summarized "all four" existing prospective studies conducted on sunscreens as minimizing premature aging, actinic keratosis and squamous cell carcinoma, but had little to no benefits for basal cell carcinoma or melanoma.

\section{Reason 6}

The law demands it! It is not optional for a company to decide to test or not test a product for human safety and/or efficacy ... it is required by law.

Perhaps the last reason one should know why this testing should be done, is that the Food Drug \& Cosmetic Act (since 1938) has placed the responsibility on industry to make sure a product is safe and effective "before" it is introduced into the marketplace; FDA's role is to review the data and determine if it is safe for human use based on the guidelines established [13]. If IARC's concerns about sunscreens were addressed back in 2000, when it was first realized that sunscreens were not as effective as thought against fighting skin cancer, we would have had almost 2 decades to develop/test new sunscreen actives and perhaps by now we would be discussing how these new actives significantly decreased the global incidence of skin cancer. The only question left is whether industry will conduct the FDA requested studies and prove the safety of oxybenzone and the 11 other sunscreen actives in question or will they walk away with the money made and not look back?

In summary, we argue that there are many reasons why oxybenzone needs to be evaluated for its mutagenicity, carcinogenicity and developmental/reproductive toxicities. It is a chemical that demonstrates a great deal of toxicological impact that creates harm to the environment and to ourselves. It should also be noted that oxybenzone is not the only sunscreen chemical with a significant amount of published toxicological data available. For example, in the toxicological review sent to FDA [3], there are 135+ publications on octinoxate, $90+$ publications on octocrylene... etc. The fact that the average professional no longer has enough time to read all of the research is industry's biggest benefit. It enables 
K-street lobbyists, marketing specialists and sales representatives to say whatever they want causing us to mislead the people coming to us for help. The US rate of skin cancer is closing in on 5 million cases a year with a treatment cost of $\$ 8.1$ billion annually [14], causing nearly 10,000 deaths annually in the US alone [15]. The phrase, "sunscreen saves lives", commonly used by some lobbyists and company representatives appears to be no different than the phrase "opioids are a safe and a non-additive way to treat chronic pain"!

\section{References}

1. Department of Health and Human Services Food \& Drug Administration. Sunscreen Drug Products for Over-theCounter Human Use. Federal Register. 2019; 84: 6204-6275.

2. Matta MK, Zusterzeel R, Pilli NR, et al. Effect of Sunscreen Application Under Maximal Use Conditions on Plasma Concentration of Sunscreen Active Ingredients A Randomized Clinical Trial. JAMA. 2019; 321: 2082-2091.

3. https://www.regulations.gov/document? D=FDA1978-N-0018-1508

4. French J.E. NTP technical report on the toxicity studies of 2-Hydroxy-4-methoxybenzophenone (CAS No. 131-57-7) Administered Topically and in Dosed Feed to F344/N Rats and B6C3F1 Mice. Toxic Rep Ser. 1992; 21: 1-52.

5. Rhodes MC, Bucher JR, Peckham JC, et al. Carcinogenesis studies of benzophenone in rats and mice. Food Chem Toxicol. 2007; 45: 843-851.

6. Department of Health and Human Services Food \& Drug
Administration. Food Additive Regulations; Synthetic Flavoring Agents and Adjuvants. Federal Register. 2018; 83: 50490-50503.

7. http://publications.iarc.fr/_publications/media/download/3129/12caa438d0a8ebad02c51f7c5c8328b850b09a35. pdf

8. https://oehha.ca.gov/proposition-65/chemicals/benzophenone

9. Wilson RD. Principles of human teratology: drug, chemical, and infectious exposure. J Obstet Gynaecol Can. 2007; 29: 911-917.

10. Bos JD, Meinardi MMHM. The 500 Dalton rule for the skin penetration of chemical compounds and drugs. Exp Dermatol. 2000; 9: 165-169.

11. Vainio H, Miller AB, Bianchini F. An international evaluation of the cancer-preventive potential of sunscreens. Int J Cancer. 2000; 88: 838-842.

12. Waldman RA, Grant-Kels JM. The role of sunscreen in the prevention of cutaneous melanoma and nonmelanoma skin cancer. J Am Acad Dermatol. 2019; 80: 574-576.

13. https://www.fda.gov/regulatory-information/search-fdaguidance-documents/nonprescription-sunscreen-drugproducts-safety-and-effectiveness-data

14. Guy Jr GP, Machlin SR, Ekwueme DU, et al. Prevalence and costs of skin cancer treatment in the U.S., 2002-2006 and 2007-2011. Am J Prev Med. 2015; 48: 183-187.

15. Siegel RL, Miller KD, Jemal A. Cancer statistics, 2018. CA Cancer J Clin. 2018; 68: 7-30. 